\title{
Photonic crystals with anomalous dispersion: Unconventional propagating modes in the photonic band gap
}

\author{
Daniel Hermann, Marcus Diem, ${ }^{*}$ and Sergei F. Mingaleev ${ }^{\dagger}$ \\ Institut für Theorie der Kondensierten Materie, Universität Karlsruhe, 76128 Karlsruhe, Germany \\ and Institut für Theoretische Festkörperphysik, Universität Karlsruhe, 76128 Karlsruhe, Germany \\ Antonio García-Martín ${ }^{\ddagger}$ and Peter Wölfle \\ Institut für Theorie der Kondensierten Materie, Universität Karlsruhe, 76128 Karlsruhe, Germany \\ Kurt Busch \\ Institut für Theorie der Kondensierten Materie, Universität Karlsruhe, 76128 Karlsruhe, Germany; \\ Department of Physics and College of Optics and Photonics: CREOL and FPCE, \\ University of Central Florida, Orlando, Florida 32816, USA; \\ and Institut für Theoretische Festkörperphysik, Universität Karlsruhe, 76128 Karlsruhe, Germany
}

(Received 24 September 2007; published 11 January 2008)

\begin{abstract}
We present an investigation of the optical properties of photonic crystals whose constituent materials exhibit anomalous dispersive behavior. In particular, the anomalous dispersion near resonances may lead to additional propagating modes in the gap of the undoped system for a localized region of wave-vector space. Such a system may be realized by infiltrating quantum dots in polymer suspensions into the pores of two-dimensional high-index photonic crystals. An evaluation of the absorption lengths associated with these unconventional modes and corresponding transmission calculations demonstrate that this effect can be observed in currently accessible structures.
\end{abstract}

DOI: 10.1103/PhysRevB.77.035112

PACS number(s): 42.70.Qs, 42.25.Bs

\section{INTRODUCTION}

Progress in photonics is closely related to development of optical materials with tailor-made properties. Photonic crystals $(\mathrm{PCs})^{1-3}$ represent a novel class of man-made optical materials. The judicious design of these two-dimensional (2D) or three-dimensional (3D) periodic dielectric arrays allows one to tailor the photonic dispersion relation and the corresponding mode structure to almost any need. In particular, the flexibility associated with material composition, lattice periodicity, and symmetry together with the deliberate creation of defect structures makes PCs the optical analog of an electronic semiconductor.

The usefulness of PCs and defect structures embedded in them may be substantially enhanced if the structures exhibit one or more forms of tunability. This has led a number of authors to propose PC structures whose constituent materials exhibit tunable properties such as temperature-dependent refractive indices, ${ }^{4}$ strongly dispersive behavior, ${ }^{5}$ as well as electro- ${ }^{4-6}$ and magnetooptically ${ }^{7}$ controllable anisotropies.

In this paper, we consider photonic band structures and absorption lengths of PCs whose constituent materials exhibit anomalous dispersion. By embedding these materials into a PC, one may expect to modify the photonic band structure considerably, if the resonance frequency is tuned to lie close to a photonic band edge. As we will show, the hybridization of the electromagnetic wave mode with the local dielectric modes leads to a splitting of the wave mode into three submodes in a limited region of wave-vector space. Of these three modes, two are stable and form a bubble shape in the band structure. However, as any frequency dependence of the effective dielectric constant is necessarily accompanied by a dissipative component, the question arises as to whether these bubble modes are overdamped. To answer this question, it requires a full treatment of the propagating and absorptive properties of the electromagnetic wave. As a consequence, we describe a highly efficient on-shell methodology based on photonic Wannier functions, ${ }^{8}$ which allows us to solve for the photonic band structure and absorption lengths associated with complex-valued and frequency-dependent dielectric constants. We present a careful study of this problem and conclude that the bubble modes should, indeed, be observable in currently accessible materials.

The paper is organized as follows. In Sec. II, we define the model system used in the following sections. The numerical methods for band structure and attenuation length calculations of PCs with dispersive components are described and discussed in Sec. III. In Sec. IV, we present results for the model system, discuss the effects of the anomalous dispersion of the constituent materials on the photonic band structure, and investigate the attenuation length resulting from the nonzero imaginary part of the dielectric constant. Finally, we summarize our results in Sec. V.

\section{MODEL SYSTEM}

In the following sections, we discuss in detail the case of TM-polarized radiation in 2D macroporous silicon $\mathrm{PCs}^{9}$ (square lattice of pores with lattice constant $a$ in silicon, dielectric constant of silicon $\varepsilon_{\mathrm{Si}}=12.0$, and electric field polarized parallel to the pore axis), where the pores (radius $r / a=0.475$ ) have been infiltrated with a polymer (typical di- 


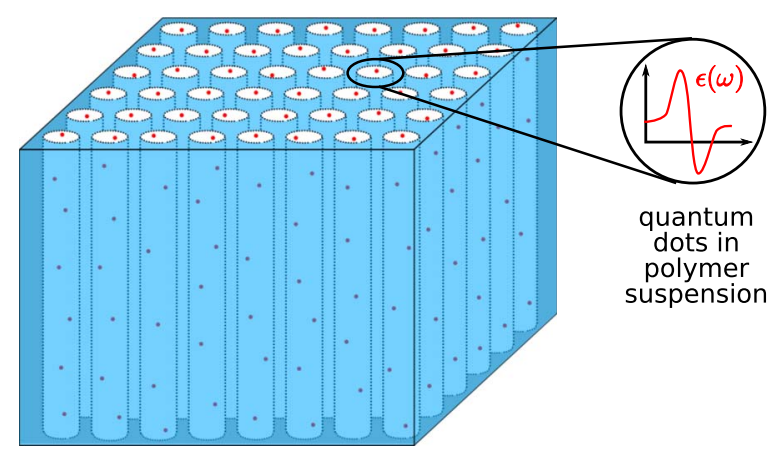

FIG. 1. (Color online) Model system of a 2D macroporous silicon PC infiltrated with quantum dots in a polymer suspension. This PC consists of a 2D macroporous silicon backbone (dielectric constant $\varepsilon_{\mathrm{Si}}=12.0$ ) into which a square lattice of pores (radius $r / a$ $=0.45$ ) has been etched, which subsequently have been filled with a low-index polymer (dielectric constant $\varepsilon_{\text {polymer }}=2.56$ ).

electric constant $\left.\varepsilon_{\text {polymer }}=2.56\right)$ that contains a (small) volume fraction of quantum dots. This is schematically depicted in Fig. 1, and we would like to note that similar systems have recently been realized, ${ }^{10,11}$ albeit for different purposes. However, we would like to emphasize that all our arguments are directly applicable to the case of TE-polarized radiation in 2D PCs as well as to the case of 3D PCs if only the constituent materials exhibit frequency ranges with anomalous dispersion.

In our simplified approach, the quantum dots are modeled through a (linear) two-level dielectric constant,

$$
\varepsilon_{\mathrm{tl}}(\omega)=1+\frac{\omega_{p}^{2}}{\omega_{0}^{2}-\omega^{2}-i \gamma \omega},
$$

where $\omega_{0}, \omega_{p}$, and $\gamma$ denote, respectively, the resonance frequency, the oscillator strength, and the linewidth (damping constant). Owing to their small size of a few nanometers relative to the optical wavelength, we may treat the admixture of a certain concentration $\eta$ of quantum dots to the polymer within a Maxwell-Garnett effective dielectric constant approach. ${ }^{12}$ As a consequence, the effective dielectric constant of this doped polymer that fills the pores of the macroporous silicon PCs reads

$$
\varepsilon_{\text {pore }}(\omega)=\varepsilon_{m}\left(1+\frac{3 \eta \alpha(\omega)}{1-\eta \alpha(\omega)}\right) .
$$

Here, we have introduced the polarizability $\alpha(\omega)$ of the quantum dots within the polymer matrix $\left(\varepsilon_{m}=\varepsilon_{\text {polymer }}\right)$ according to

$$
\alpha(\omega)=\frac{\varepsilon_{t l}(\omega)-\varepsilon_{m}}{\varepsilon_{t l}(\omega)+2 \varepsilon_{m}} .
$$

In Fig. 2, we depict the frequency dependence of the real and imaginary parts of this effective dielectric constant [Eq. (2)] for a resonance frequency $\omega_{0} a / 2 \pi c=0.245$, oscillator strength $\omega_{p}=0.8 \omega_{0}$, damping constant $\gamma=0.01 \omega_{0}$, and several realistic values of the concentration $\eta$. In fact, changing the concentration $\eta$ of quantum dots allows a rather simple way

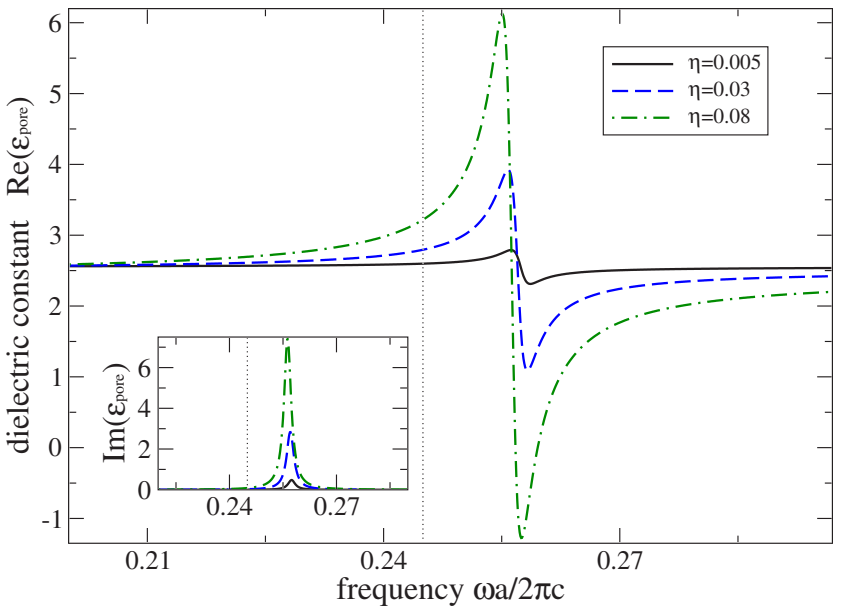

FIG. 2. (Color online) Effective dielectric constant of a typical polymer doped with quantum dots for three different concentrations $\eta$. The quantum-dot parameters are given in the text. The resonance of this Maxwell-Garnett effective dielectric constant is shifted relative to the resonance frequency of isolated quantum dots (vertical dotted line).

of significantly tuning the pore dielectric constant in the vicinity of the quantum-dot resonance frequency. As a result of the hybridization of the quantum-dot modes with the background modes provided by the polymer, this resonance is slightly shifted relative to the bare quantum-dot resonance frequency.

Before proceeding to photonic band structure computations of composite PC structures, we would like to make two observations. First, while we have derived the effective dielectric constant $\varepsilon_{\text {pore }}(\omega)$ of the pore material for a specific and easily realizable model system (see Ref. 11), it should be clear that its frequency-dependent behavior depicted in Fig. 2 is rather generic and can be obtained via many other routes such as the growth of polar semiconductor nanocrystals within the pores. Second, the value of the resonance frequency determines the PC's photonic bands which experience the effects of the anomalous dispersion depicted in Fig. 2 most strongly. Therefore, the undoped PC has to be carefully engineered in order to ensure that the corresponding Bloch functions sample the pores sufficiently well. Analogous considerations apply to other structures. We will return to this issue in Sec. IV.

\section{BAND STRUCTURE AND ATTENUATION LENGTH COMPUTATIONS}

The Maxwell equations for time-harmonic TM-polarized light propagating in the $(x, y)$ plane of a 2D PC can be reduced to a scalar wave equation for the $z$-component $E(\vec{r})$ of the electric field.

$$
\nabla^{2} E(\vec{r})+\left(\frac{\omega}{c}\right)^{2} \varepsilon_{\mathrm{pc}}(\omega, \vec{r}) E(\vec{r})=0 .
$$

Here, $\nabla^{2}=\partial_{x}^{2}+\partial_{y}^{2}$ and $c$ denote the 2D Laplacian and the vacuum speed of light, respectively. The dielectric constant 
$\varepsilon_{\mathrm{pc}}(\omega, \vec{r}) \equiv \varepsilon_{\mathrm{pc}}(\omega, \vec{r}+\vec{R})$ contains all the information about the PC and is periodic with respect to the set $\mathcal{R}=\left\{n_{1} \vec{a}_{1}\right.$ $\left.+n_{2} \vec{a}_{2} ;\left(n_{1}, n_{2}\right) \in \mathcal{Z}^{2}\right\}$ of lattice vectors $\vec{R}$ that are generated by the primitive translations $\vec{a}_{1}$ and $\vec{a}_{2}$. For our model system, the PC dielectric constant reads as

$$
\varepsilon_{\mathrm{pc}}(\omega, \vec{r})=\varepsilon_{\mathrm{Si}}+\left[\varepsilon_{\text {pore }}(\omega)-\varepsilon_{\mathrm{Si}}\right] \sum_{\vec{R}} \Theta(r-|\vec{r}-\vec{R}|),
$$

where we have introduced the Heaviside function $\Theta(x)$.

Equation (4) represents a differential equation with periodic coefficients and, therefore, its solutions obey the BlochFloquet theorem,

$$
E_{n \vec{k}}\left(\vec{r}+\vec{a}_{j}\right)=e^{i \vec{k} \vec{a}_{j}} E_{n \vec{k}}(\vec{r}), \quad j=1,2 .
$$

In the reduced zone scheme, ${ }^{13}$ which we adopt here, the Bloch modes $E_{n \vec{k}}(\vec{r})$ are labeled with the composite index $(n \vec{k})$, where $n$ and $\vec{k}$, respectively, denote the band index and the wave vector in the first Brillouin zone (BZ).

In the case of a frequency-independent dielectric constant, $\varepsilon_{\mathrm{pc}}(\omega, \vec{r}) \equiv \varepsilon_{\mathrm{pc}}(\vec{r})$, Eq. (4) can be solved as a standard eigenvalue problem using general purpose methodologies such as plane wave expansion (PWE) approaches, ${ }^{14,15}$ finitedifference based multigrid methods, ${ }^{16}$ or advanced finite element techniques. ${ }^{17}$ However, the situation becomes much more complicated for frequency-dependent dielectric constants which we study in this paper. In this case, Eq. (4) constitutes a nonlinear elliptic eigenvalue problem with Bloch-boundary conditions and, therefore, solving Eq. (4) either requires the usage of an on-shell methodology, ${ }^{18,19}$ which - for a given frequency $\omega$-allows one to compute the allowed wave vectors $\vec{k}(\omega)$ or an extension of the standard band structure methods to nonlinear eigenvalue problems. For instance, the standard PWE approach has been adapted to treat specific forms of frequency-dependent dielectric constants, and, recently, it has been reformulated to facilitate on-shell computations. ${ }^{20}$ However, the Kramers-Kronig relations - which are a manifestation of causality ${ }^{21}$-require that dispersive materials have to be described through a complex-valued dielectric constant such as those described in Eqs. (1) and (2). As a consequence and in order to ensure the viability of the results, absorption has to be taken into account by all methods of photonic band structure computations.

In general, there exist two distinct ways how to deal with absorption in photonic band structure theory. Within the first route, real-valued wave vectors are maintained so that absorption leads to complex eigenfrequencies. ${ }^{22,23}$ From a mathematical point of view, this has the advantage that the Bloch modes can be normalized. Physically, this corresponds to the situation where the Bloch modes acquire a finite lifetime, i.e., an initially excited Bloch mode decays as time progresses. The realization of a corresponding experiment appears to be rather challenging. The second option is to retain real-valued frequencies and to associate the effects of absorption with complex-valued wave vectors. As a result, the Bloch modes resist normalization. On the other hand, an imaginary part of the wave vector allows one to define a length scale that describes how waves at a given frequency are attenuated within a PC. This attenuation length accounts for both the effects of absorption and photonic band gaps. The latter are also present in the nonabsorbing case. An experimental realization of this situation would have to be based on studies of the transmission through finite but sufficiently large PC samples, so that effects related to the PC surfaces (for instance, in- and outcoupling efficiencies) can be separated from the attenuation in the bulk PC (Sec. IV).

The relation between the two approaches discussed above is nontrivial. For instance, in the case of complex eigenfrequencies as functions of real wave vectors, it would be necessary to develop an analytical continuation of those frequencies to complex-valued wave vectors and to search for wave vectors for which the imaginary part of the frequency vanishes. Since the eigenfrequencies are, in general, only known numerically, this is a formidable task. Furthermore, even in the weakly absorbing case, simple perturbation techniques for the transition from complex frequencies to complex wave vectors fail,${ }^{22}$ although recent advances ${ }^{23}$ suggest that more sophisticated approaches could improve the situation (see also Ref. 24).

In the following sections, we describe three independent and somewhat complementary methods that allow one to develop a complete physical picture of PCs with dispersive constituent materials and apply those methods to our model problem with anomalous dispersion. First, we present an efficient on-shell methodology based on photonic Wannier functions that allows one to carry out band structure and attenuation length calculations for general frequencydependent and complex-valued dielectric constants. We then provide a brief summary of an extension of standard band structure calculation techniques (initially described in Ref. 24 and subsequently rediscovered in Ref. 25) that allows one to deal with frequency-dependent but real-valued dielectric constants. This extension may provide the basis for reasonable approximations in systems where absorption can be treated perturbatively. ${ }^{24}$ Most importantly, this extension suggests a straightforward geometrical interpretation and additional intuitive insights into the results obtained from the more general Wannier function approach. Finally, we demonstrate how attenuation lengths can be extracted from transmission calculations through finite PC samples. These calculations are based on a rigorous coupled wave analysis (RCWA) approach with numerical scattering matrices and allow a direct comparison with the Wannier function results for infinite PCs.

\section{A. Wannier function approach}

In order to solve Eq. (4) within the Wannier function approach, we decompose the complex-valued PC dielectric constant $\varepsilon_{\mathrm{pc}}(\omega, \vec{r})$ into a real-valued and nondispersive reference part $\varepsilon_{\text {ref }}(\vec{r})$ and a complex-valued frequency-dependent part $\Delta \varepsilon(\omega, \vec{r})=\varepsilon_{\mathrm{pc}}(\omega, \vec{r})-\varepsilon_{\mathrm{ref}}(\vec{r})$ that both retain the lattice periodicity of the PC. As alluded to above, for the reference system, we can easily solve the corresponding wave equation, 


$$
\nabla^{2} E(\vec{r})+\left(\frac{\omega}{c}\right)^{2} \varepsilon_{\text {ref }}(\vec{r}) E(\vec{r})=0,
$$

via standard band structure computations. Moreover, we have a certain flexibility in choosing the reference dielectric constant $\varepsilon_{\text {ref }}(\vec{r})$. The actual choice of $\varepsilon_{\text {ref }}(\vec{r})$ should be guided by physical considerations, such as the range of values, which the PC's different constituent material dielectric constants take on over the frequency range of interest so that the reference dielectric constant is (in some sense) not too far from the actual system that should be solved. For our model system, the natural choice for $\varepsilon_{\text {ref }}(\vec{r})$ is the 2D macroporous silicon PC whose pores have been infiltrated with the undoped polymer.

As a result, Eq. (7) provides us with a reference band structure and corresponding reference Bloch functions $E_{n \vec{k}}^{(0)}(\vec{r})$. These Bloch functions are then further processed to yield the reference PC's photonic Wannier functions,

$$
W_{n \vec{R}}(\vec{r})=\frac{V_{\mathrm{WSC}}}{(2 \pi)^{2}} \int_{\mathrm{BZ}} d^{2} k e^{-i \vec{k} R} \sum_{m} U_{n m}(\vec{k}) E_{n \vec{k}}^{(0)}(\vec{r})
$$

Here, $V_{\text {WSC }}$ denotes the volume of the PC's Wigner-Seitz cell. The unitary matrices $U_{n m}(\vec{k})$ have to be determined numerically and facilitate that the Wannier functions are maximally localized in the unit cell that corresponds to the lattice vector $\vec{R}$. Owing to the fact that photonic Wannier functions are essentially the result of a lattice Fourier transform of Bloch functions, they have encoded into them the entire information of the reference PC's photonic band structure and exhibit very useful orthonormality and translational properties,

$$
\begin{gathered}
\int d^{2} r W_{m \vec{R}}^{*}(\vec{r}) \varepsilon_{\mathrm{ref}}(\vec{r}) W_{m^{\prime} R^{\prime}}(\vec{r})=\delta_{m m^{\prime}} \delta_{R R^{\prime}}^{\vec{\prime}}, \\
W_{m \vec{R}}(\vec{r})=W_{m 0}(\vec{r}-\vec{R}) .
\end{gathered}
$$

In Eq. (9), the integration extends over all space and $\delta_{m m^{\prime}}$ denotes the Kronecker symbol. For details on the actual construction of photonic Wannier functions and their usage in the design of functional elements, we refer to Ref. 8. In Figs. 3 and 4, we display, respectively, the photonic band structure (calculated with the multigrid method ${ }^{16}$ ) and a selection of maximally localized Wannier functions for the reference PC structure. The band structure exhibits a photonic band gap in the frequency range $\omega a / 2 \pi c \in[0.233,0.249]$ located between bands 1 and 2. For the subsequent computations, we employ the first 18 Wannier functions.

These reference Wannier functions may now be utilized for solving the photonic band structure of the full system with anomalous dispersion,

$$
\nabla^{2} E(\vec{r})+\left(\frac{\omega}{c}\right)^{2}\left[\varepsilon_{\mathrm{ref}}(\vec{r})+\Delta \varepsilon(\omega, \vec{r})\right] E(\vec{r})=0 .
$$

Upon inserting an expansion of the electric field into Wannier functions,

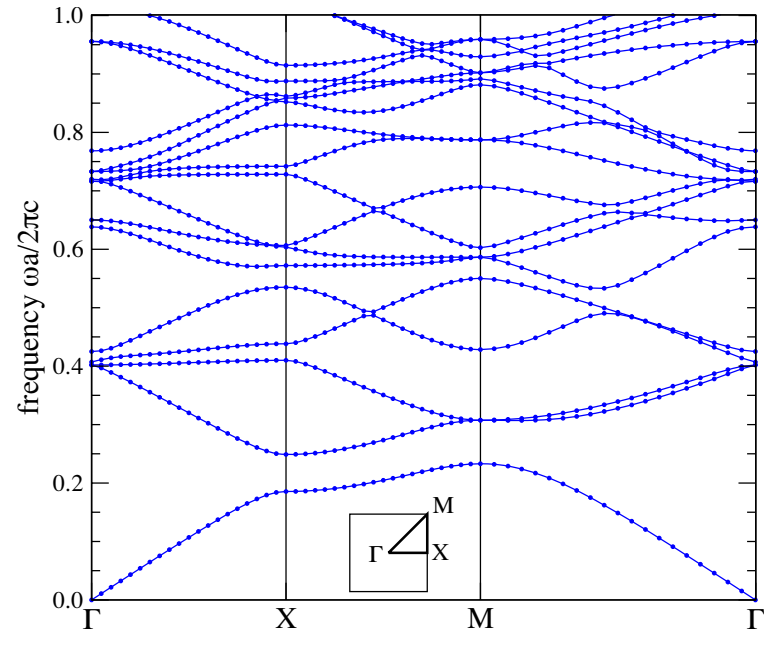

FIG. 3. (Color online) Photonic band structure for TM-polarized radiation propagating in the reference PC structure. The associated system parameters are listed in the caption of Fig. 1.

$$
E(\vec{r})=\sum_{m, \vec{R}} E_{m \vec{R}} W_{m \vec{R}}(\vec{r})
$$

we obtain-via projecting onto the same basis-a matrix equation for the unknown amplitudes $E_{m \vec{R}}$ that in component form reads as

$$
\sum_{m^{\prime}, \vec{R}^{\prime}} M_{\vec{R} R^{\prime}}^{m m^{\prime}}(\omega) E_{m^{\prime} \vec{R}^{\prime}}=0
$$

with the corresponding frequency-dependent system matrix,

$$
M_{\vec{R} R^{\prime}}^{m m^{\prime}}(\omega)=\delta_{m m^{\prime}} \delta_{R R^{\prime}}^{\vec{\prime}}+D_{\overrightarrow{R R^{\prime}}}^{m m^{\prime}}(\omega)-\left(\frac{c}{\omega}\right)^{2} A_{\overrightarrow{R R^{\prime}}}^{m m^{\prime}} .
$$

Here, the entries are constructed from the overlap matrix elements of Wannier functions with respect to the operators appearing in Eq. (11), utilizing the orthonormality of the Wannier functions [Eq. (9)]. Explicitly, we have

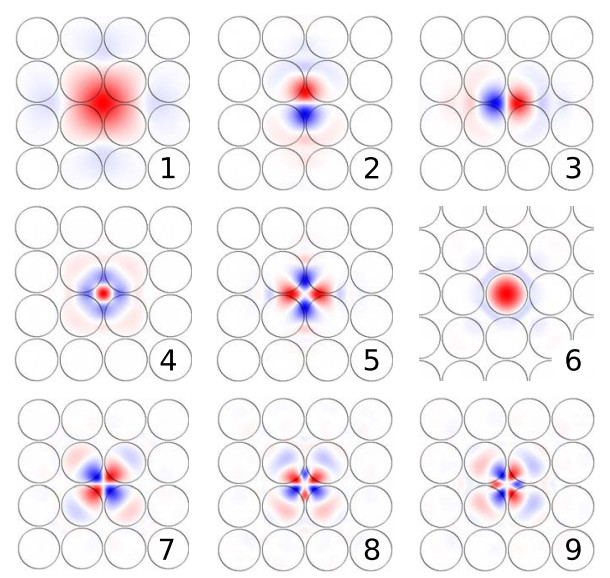

FIG. 4. (Color online) A selection of maximally localized Wannier functions for the reference PC structure. The corresponding photonic band structure is displayed in Fig. 3 and the associated system parameters are listed in the caption of Fig. 1. 


$$
\begin{gathered}
A_{\vec{R} R^{\prime}}^{m m^{\prime}}=-\int d^{2} r W_{m \vec{R}}^{*}(\vec{r}) \nabla^{2} W_{m^{\prime} R^{\prime}}(\vec{r}), \\
D_{\vec{R} \vec{R}^{\prime}}^{m m^{\prime}}(\omega)=\int d^{2} r W_{m \vec{R}}^{*}(\vec{r}) \Delta \varepsilon(\omega, \vec{r}) W_{m^{\prime} R^{\prime}}(\vec{r}) .
\end{gathered}
$$

With the help of the translational properties of both the Wannier functions [Eq. (10)] and $\Delta \varepsilon(\omega, \vec{r})$, it is straightforward to show that the system matrix itself exhibits translational symmetries,

$$
M_{\vec{R}-\vec{a}_{i}, \vec{R}^{\prime}-\vec{a}_{i}}^{m m^{\prime}}(\omega)=M_{\vec{R}, \vec{R}^{\prime}}^{m m^{\prime}}(\omega),
$$

with respect to both lattice vectors $\vec{a}_{i}$, where $i=1,2$. We are interested in the Bloch mode solutions of our model system including the material dispersion, i.e., the Bloch modes $E_{n \vec{k}}(\vec{r})$ of Eq. (11) or its representation within the Wannier function approach [Eq. (13)]. As alluded to above, this may be facilitated within an on-shell approach. Therefore, we fix the real frequency $\omega$, choose a direction $\hat{k}$ in wave-vector space, and determine the allowed complex-valued magnitudes $k$ of the full wave vector $\vec{k}=k \hat{k}$ so that their real parts lie within the first BZ.

This is realized through several steps. First, we determine suitable integer numbers $\alpha$ and $\beta$ such that the reciprocal lattice vector $\vec{G}=\alpha \vec{b}_{1}+\beta \vec{b}_{2}$ is parallel to $\hat{k}$. As usual, the reciprocal basis vectors $\vec{b}_{j}$, where $j=1,2$ are defined through their orthogonality condition $\left(\vec{a}_{i} \cdot \vec{b}_{j}\right)=2 \pi \delta_{i j}$ relative to the real space lattice vectors $\vec{a}_{i}$, with $i=1,2$.

Second, we define the real space lattice vectors $\vec{s}_{\|}=\alpha \vec{a}_{1}$ $+\beta \vec{a}_{2}$ and $\vec{s}_{\perp}=\alpha \vec{a}_{2}-\beta \vec{a}_{1}$ that define the sides of the computational supercell employed in the on-shell computations. In addition, we define a set of $M$ lattice vectors $\vec{\rho}_{\ell}$, which consists of the origin $\vec{\rho}_{1}=\overrightarrow{0}$ and all the sites $\vec{\rho}_{\ell}$, with $\ell$ $=2, \ldots, M$, inside the supercell formed by the vectors $\vec{s}_{\|}$and $\vec{s}_{\perp}$. As a consequence, any lattice site $\vec{R}$ of the PC can uniquely be expressed as

$$
\vec{R} \equiv \vec{R}_{i j \ell}=i \cdot \vec{s}_{\|}+j \cdot \vec{s}_{\perp}+\vec{\rho}_{\ell}
$$

such that the three integers $i, j$, and $\ell$ determine a partitioning of the PC into supercells. This is illustrated in Fig. 5. This partitioning facilitates a rewriting of Eq. (13) as

$$
\sum_{m^{\prime}, i^{\prime}, j^{\prime}, \ell^{\prime}} M_{\vec{R}_{i j \ell} m^{\prime} \vec{R}_{i^{\prime} j^{\prime} \ell^{\prime}}}^{\prime}(\omega) E_{m^{\prime} R_{i^{\prime} j^{\prime} \ell^{\prime}}}=0, \quad \forall i, j, m, \ell .
$$

By construction, the Bloch-Floquet theorem [Eq. (6)] stipulates a periodic boundary condition on the supercell in the direction of $\vec{s}_{\perp}$; i.e., we have $E_{n \vec{k}}\left(\vec{r}+j \cdot \vec{s}_{\perp}\right) \equiv E_{n \vec{k}}(\vec{r})$. Therefore, we obtain that $E_{m \vec{R}_{i j \ell}}=E_{m \vec{R}_{i 0 \ell}}$ for all integers $j$.

Based on the above considerations and upon taking into account Eq. (17), we may rewrite Eq. (19) in a matrix form that is suitable for band structure computations,

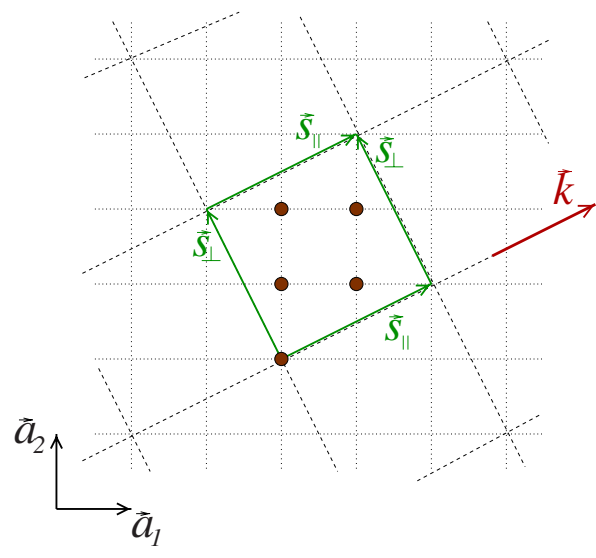

FIG. 5. (Color online) Illustration of the partitioning of real space in order to facilitate an on-shell band structure calculation. For a fixed frequency $\omega$ and direction $\hat{k}$ in reciprocal space, we partition the PC into identical rectangular supercells whose sides are given by $\vec{s}_{\|}=\alpha \vec{a}_{1}+\beta \vec{a}_{2}$ and $\vec{s}_{\perp}=\alpha \vec{a}_{2}-\beta \vec{a}_{1}$, where $\alpha$ and $\beta$ are integers such that $\vec{G}=\alpha \vec{b}_{1}+\beta \vec{b}_{2}$ is parallel to $\hat{k}$. Periodic and Blochboundary conditions are, respectively, applied along $\vec{s}_{\perp}$ and $\vec{s}_{\|}$. For this illustration on a square lattice, we have chosen $\alpha=2$ and $\beta=1$, so that a supercell contains five unit cells of the PC.

$$
\sum_{i^{\prime}=-\infty}^{\infty} \hat{\mathcal{M}}_{i-i^{\prime}}(\omega) \overrightarrow{\mathcal{E}}_{i^{\prime}}=0, \quad \forall i
$$

Here, we have introduced the vectors $\overrightarrow{\mathcal{E}}_{i}$ and submatrices $\hat{\mathcal{M}}_{i}$ according to

$$
\begin{gathered}
\overrightarrow{\mathcal{E}}_{i}=\left\{E_{m, \vec{R}_{i 0 \ell},} \quad \forall m, \ell\right\}, \\
\hat{\mathcal{M}}_{i}=\left\{\sum_{j=-\infty}^{\infty} M_{\vec{R}_{i j \ell}^{m m^{\prime}} \vec{R}_{00 \ell^{\prime}},}, \quad \forall m, m^{\prime}, \ell, \ell^{\prime}\right\} .
\end{gathered}
$$

In a numerical implementation, we have to truncate these infinite vectors $\overrightarrow{\mathcal{E}}_{i}$ and submatrices $\hat{\mathcal{M}}_{i}$ by taking into account a finite number $N_{W}$ of bands (or equivalently Wannier functions), i.e., $m=1, \ldots, N_{W}$ in Eqs. (20)-(22), and a finite number $L$ of coupled supercells, i.e., replacing $\infty$ in Eqs. (20) and (22) by $L$.

Following the approach of Ref. 8-originally developed for calculations of waveguide dispersion relations in $\mathrm{PCs}$ - we can now rewrite Eq. (20) in a transfer-matrix form by defining the composite vectors $\vec{F}_{i}=\left\{\overrightarrow{\mathcal{E}}_{i}, \overrightarrow{\mathcal{E}}_{i+1}, \ldots, \overrightarrow{\mathcal{E}}_{i+2 L-1}\right\}$. As a result, we obtain

$$
\vec{F}_{i-1}=\hat{T}(\omega) \vec{F}_{i}
$$

where the transfer matrix $\hat{T}(\omega)=\left\{\hat{T}_{i, j}(\omega)\right\}$ has nonzero submatrix entries of size $M * N_{W} \times M * N_{W}$ only for

$$
\hat{T}_{1, j}(\omega)=-\hat{\mathcal{M}}_{L}^{-1}(\omega) \hat{\mathcal{M}}_{L-j}(\omega) \text { for } j=1,2, \ldots, 2 L \text {, }
$$




$$
\hat{T}_{j+1, j}=\hat{1} \quad \text { for } j=1,2, \ldots, 2 L-1 .
$$

Here, we have denoted the $M * N_{W} \times M * N_{W}$ unit matrix by $\hat{1}$.

Finally, the combination of Eq. (23) and the BlochFloquet theorem [Eq. (6)] allows us-at the given frequency $\omega$ and for the given direction $\hat{k}$ - to identify the Bloch modes with wave vector $\vec{k}=k \hat{k}$ as the solutions $\vec{F}^{(n)}(\omega)$, where $n$ $=1, \ldots, 2 L * M * N_{W}$ of the transfer-matrix eigenvalue problem,

$$
\hat{T}(\omega) \vec{F}^{(n)}(\omega)=\exp \left\{\mathrm{i} \vec{k}_{n}(\omega) \cdot \vec{s}_{\|}\right\} \vec{F}^{(n)}(\omega),
$$

with the required wave vectors $\vec{k}_{n}(\omega)=k_{n}(\omega) \hat{k} \| \vec{G}$.

These solutions exist for all frequencies. In the presence of material absorption and/or for frequencies inside a stop band in the direction of $\hat{k}$, the wave vector $\vec{k}$ will be complex valued. Therefore, this complex dispersion relation $\vec{k}(\omega)$ contains the full band structure information, i.e., information on dispersion and attenuation or absorption inside the PCs.

The accuracy of this Wannier function approach can easily be tested with the help of the reference band structure. In particular, a comparison of the results of Eq. (26) with $a b$ initio computations of the reference band structure allows one to assess the number of Wannier functions $N_{W}$ and the maximal number of coupled supercells $L$ required for obtaining accurate results for the full system with dispersive constituent materials [Eq. (11)]. For instance, for wave-vector directions lying along the $\Gamma-X$ direction of the model PC, the computational supercell contains $M=1$ unit cells. In addition, we require $N_{W}=18$ Wannier functions and $L=4$ coupled supercells, so that the resulting transfer matrices $\hat{T}$ to be diagonalized are only $144 \times 144$ in size in order to obtain converged results. This is considerably less than what is required with other on-shell methods.

\section{B. Extension of standard band structure methods}

Standard band structure techniques ${ }^{14-16}$ that operate with fixed real-valued wave vectors $\vec{k}$ can be extended to deal with the nonlinear eigenvalue problem of Eq. (4) for frequency-dependent real-valued dielectric constants. This is facilitated by performing computations for a certain set of standard eigenvalue problems for frequency-independent dielectric constants and a subsequent postprocessing of the corresponding data in order to solve the nonlinear eigenvalue problem via an appropriate fix-point iteration.

Specifically, for our model problem, we identify a frequency range of interest and determine the associated range of variation of the pore dielectric constant's real part over this range. Next, we perform standard band structure computations for a set of (fictitious) frequency-independent realvalued pore dielectric constants $\varepsilon_{\text {fict }}$, which covers this range. As a result, we obtain—for the fixed wave vector $\vec{k}$-a series of "frequency lines" $\omega_{i}\left(\varepsilon_{\text {fict }}\right)$, which reflect the variation of eigenfrequencies of the individual bands (indexed through the integer $i$ ) when this (fictitious) pore dielectric constant $\varepsilon_{\text {fict }}$ varies. Then, we obtain the physical eigenfrequencies we

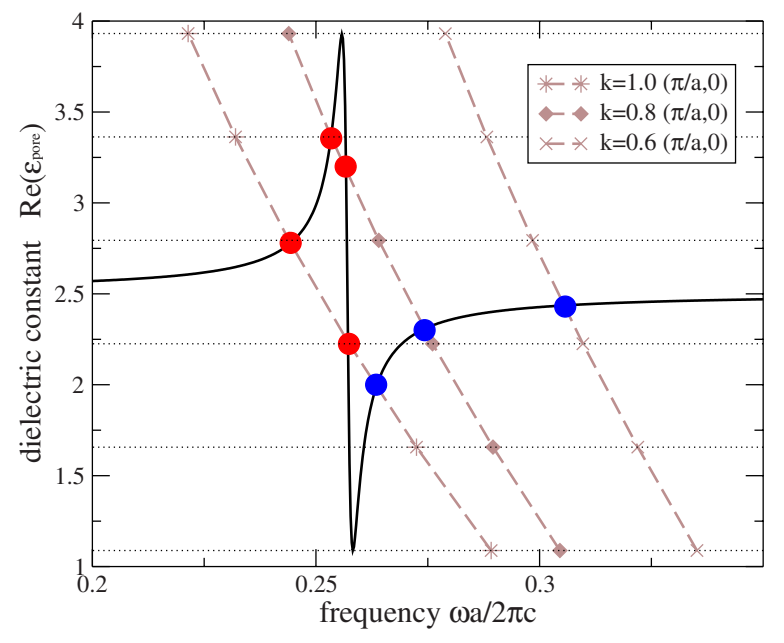

FIG. 6. (Color online) Geometric illustration of the iterative solution of the nonlinear eigenvalue problem associated with the band structure of a PC with dispersive constituent materials. The solid line depicts the frequency dependence of the real part of the model system's dielectric constant for a quantum-dot filling ratio of $\eta=0.03$. The values of the other system parameters are given in the text. The dashed lines display the frequency line $\omega_{1}\left(\varepsilon_{\text {fict }}\right)$ of the first band for three different but fixed wave vectors $\vec{k}$. The intersections of these frequency lines with the graph of the model system's dielectric constant (circles) correspond to the iterative solutions of the nonlinear eigenvalue problem of Eq. (4).

are looking for by requiring consistency between these frequency lines and the true real part of the system's dielectric constant $\varepsilon_{\text {pore }}(\omega)$. This provides us with the solutions $\omega(\vec{k})$ of the nonlinear eigenvalue problem for fixed wave vector $\vec{k}$. We may then repeat the procedure for the next wave vector.

We illustrate this fix-point iteration scheme ${ }^{24}$ in Fig. 6. The dashed lines depict the variation of the first frequency line $\omega_{1}\left(\varepsilon_{\text {fict }}\right)$ as a function of the fictitious pore dielectric constant $\varepsilon_{\text {fict }}$ for three different wave vectors in the vicinity of the $X$ point. Note that for a more transparent interpretation of the fix-point iteration, we have assigned the dependent variable $\varepsilon_{\text {fict }}$ to the ordinate. Then, the intersections (filled circles) of these lines with the frequency-dependent real part of the pore dielectric constant $\varepsilon_{\text {pore }}(\omega)$ (solid line) fulfill the consistency condition alluded to above and, therefore, represent the physical eigenfrequencies of the system.

While this fix-point iteration scheme appears to be somewhat inefficient relative to on-shell methodologies and does not directly allow one to address the issue of material absorption [imaginary part of $\varepsilon_{\mathrm{pc}}(\omega, \vec{r})$ ], it does provide an elegant geometric interpretation of the physics of PCs whose constituent materials exhibit anomalous dispersion.

In particular, Fig. 6 suggests that over certain frequency ranges with sufficiently strong anomalous material dispersion, three distinct solutions $\omega_{n}(\vec{k}), n=1,2,3$ associated with a single frequency line $\omega_{i}\left(\varepsilon_{\text {fict }}\right)$ may develop. This is in stark contrast to frequencies away from the resonance where only one solution is allowed. Owing to the facts that for finite damping, the resonance covers only a finite frequency range and that near a photonic band edge, only a finite region of 
wave-vector space is of relevance, these imply that near a photonic band edge and relative to the undoped reference system, additional propagating states may develop, which are limited to both a finite frequency and a finite wave-vector range. This will lead to the formation of a closed bubblelike object in the photonic band structure diagram of the undoped system $^{24}$ (see Fig. 9) and will be further elaborated in Sec. IV.

\section{Finite samples: Scattering-matrix approach}

An experimental investigation of PCs usually requires the measurement of reflectance and transmittance from finitesized samples. The analysis of such data may substantially benefit from a comparison with corresponding computations. For instance, reflectance and transmittance computations allow one to assess the fabricational tolerances of the structures and to assist in their design. ${ }^{26}$

In the present case, we are interested in estimating the impact of material absorption and whether any effects derived from the photonic band structure computations alluded to above could be observed experimentally. In addition, we will demonstrate below that the careful analysis of the length dependence of the transmittance through finite-sized samples at fixed frequency allows an alternative route for determining the inverse attenuation length, i.e., the imaginary part of the wave vector. The computation of the reflectance and transmittance from periodic structure is a well-documented subject of diffractive optics, ${ }^{27-29}$ where it is often referred to as the RCWA. In the context of PCs, it is more often known as the scattering-matrix method. ${ }^{30}$ For our subsequent computations, we utilize our own implementation of the scatteringmatrix method ${ }^{30}$ enhanced with the correct Fourier factorization techniques ${ }^{28,31}$ that significantly improve the convergence properties of the approach.

In Fig. 7, we display the frequency dependence of the transmittance (at normal incidence) through a finite-sized PC (our model system with quantum-dot concentration $\eta=0.03$ ) for several values of the sample thickness (measured in terms of the number of unit cells $N$ ). The sample is oriented such that normal incidence corresponds to propagation along the crystallographic $\Gamma-X$ direction. In addition, we have chosen the surface termination such that we cut right through the middle of a row of pores of the infinite PC. Clearly, in our transmittance or reflectance computations, we have refrained from filling these slit open pores with the doped polymer. In addition, we display complementary reflectance calculations for the same structure in Fig. 8 (note, however, the different scalings of the ordinates in Figs. 7 and 8).

Taken together, Figs. 7 and 8 suggest that once the PC sample exceeds a certain length (about 20 unit cells in our example and considered frequency range), the in- and outcoupling processes to and from the sample have been decoupled such that for larger sample sizes, the transmittance is only affected by the total attenuation within an effectively bulk PC. Consequently, we may determine the total attenuation length $l_{\omega}$ of the corresponding bulk PC for a fixed frequency $\omega$ by fitting the corresponding length dependence of the transmittance $T_{\omega}(N)$ to an exponential of the form

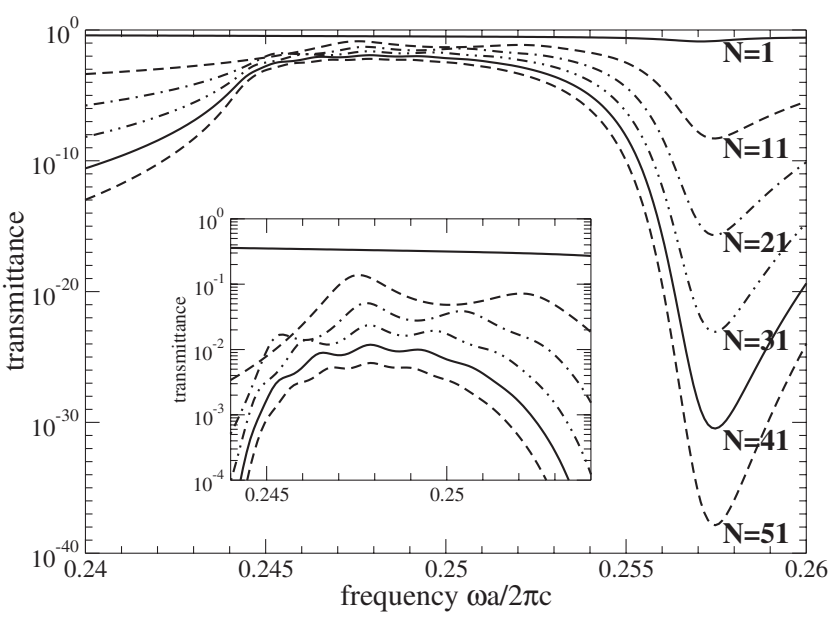

FIG. 7. Frequency dependence of the transmittance at normal incidence for finite-sized PC samples with different thicknesses $(N=1,11,21,31,41,51$ unit cells). The PC is oriented such that normal incidence corresponds to propagation along the $\Gamma-X$ direction. The inset demonstrates that once the sample thickness exceeds about 20 unit cells, the transmittance decays approximately exponentially with thickness. This behavior should be compared with the corresponding reflectance calculations displayed in Fig. 8. For actual calculations of the attenuation length, sample thicknesses in the range of $N=1, \ldots, 100$ with a step size of $\Delta N=1$ have been analyzed. The PC parameters are given in the text.

$T_{\omega}(N)=C_{\omega} \exp \left(-N a / l_{\omega}\right)$. This total attenuation length is connected to the imaginary part of the complex wave vectors' modulus in the corresponding crystallographic direction $\hat{k} \| \Gamma-X$ via $\operatorname{Im}[k(\omega)]=1 /\left(2 l_{\omega}\right)$. For the actual calculations of the attenuation length via transmittance computations, we have analyzed the transmittance values for thicknesses rang-

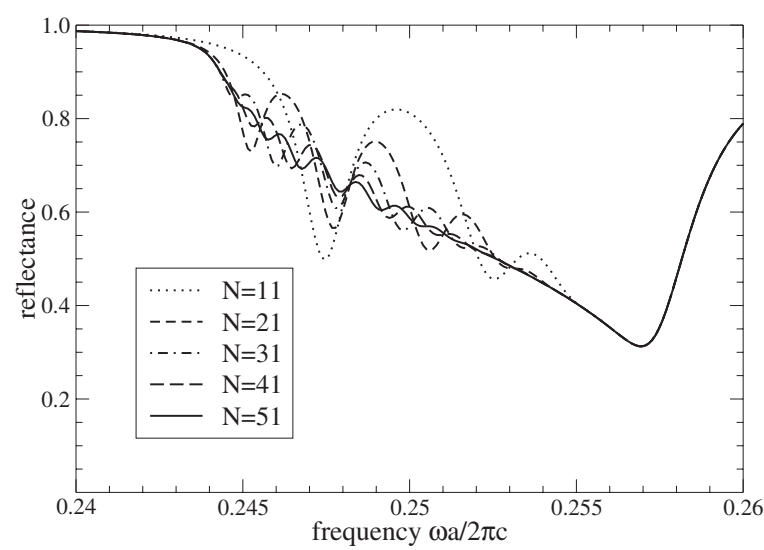

FIG. 8. Frequency dependence of the reflectance at normal incidence for finite-sized PC samples with different thicknesses $(N$ $=11,21,31,41,51$ unit cells). The PC is oriented such that normal incidence corresponds to propagation along the $\Gamma-X$ direction. Once the sample thickness exceeds about 20 unit cells, the reflectance values (approximately) become independent of the sample thickness. This behavior should be compared with the corresponding transmittance calculations displayed in Fig. 7. The PC parameters are given in the text. 
ing from 1 to 100 unit cells with a step size of 1 . More precisely, Fig. 8 shows that the reflectance does not change within a $1 \%$ margin for frequencies below $\omega a / 2 \pi c=0.243$ and above $\omega a / 2 \pi c=0.255$ for sample thicknesses of $N$ $>10$ unit cells. This behavior originates in the absorptive behavior of the material, which strongly suppresses the Fabry-Perot oscillations that could be observed for absorption-free systems. Therefore, in these frequency ranges, the exponential could be fitted to the computed data with $N>10$. However, for frequencies in between, this damping out of the Fabry-Perot oscillations is less effective and the reflectance approaches saturation only for sample thicknesses of $N>20$ unit cells. As a consequence, the exponential fit should only be carried out for the corresponding range of $N>20$. In order to be consistent, we have computed the total attenuation length for all frequencies by fitting to the data for $N>20$. In addition, we have checked that in those cases for which we could fit to larger data sets, no discrepancies occur.

\section{RESULTS}

We now apply the computational methods described in Sec. III to our model problem in Sec. II. First, the extended band structure approach (Sec. III B) will be used to derive a physical interpretation of the results. This will be followed by a fully quantitative analysis through the Wannier function technique (Sec. III A) and the scattering-matrix method (Sec. III C). The latter also allows one to assess the feasibility of corresponding experiments.

We have chosen the parameters of our model system such that the resonance of the pore dielectric constant [Eq. (2)] lies inside the photonic band gap of the undoped system, close to the upper band edge of the first band gap (see Figs. 2 and 3). This choice has been guided by the fact that the Bloch modes associated with the second band near the $X$ point have a significant part of the field inside the pores. Therefore, these modes are rather sensitive to any modification of the pore properties.

For the time being, we disregard the imaginary part in the pore dielectric constant and apply the extended band structure method to the problem with a quantum-dot concentration $\eta=0.03$. The resulting band structure is depicted in Fig. 9 and shows a significant splitting of the second band into one continuous band and a bubblelike structure of finite extent in wave-vector space located partly inside the photonic band gap of the undoped reference system.

Despite the fact that these frequencies appear inside the photonic band gap of the undoped system, the associated modes represent - in the present case of ignored material absorption-bona fide extended Bloch modes, as they do obey the Bloch-Floquet theorem. The occurrence of this bubblelike structure is a direct consequence of the anomalous dispersion over a finite frequency range provided by the "energy-dependent" potential $\varepsilon_{\mathrm{pc}}(\omega, \vec{r})$, which directly translates into both a finite extent in frequency and wave vector of the bubble. To the best of our knowledge, this does not have an analogy in electronic crystals, although a potential function with a nonmonotonic variation in energy would allow a

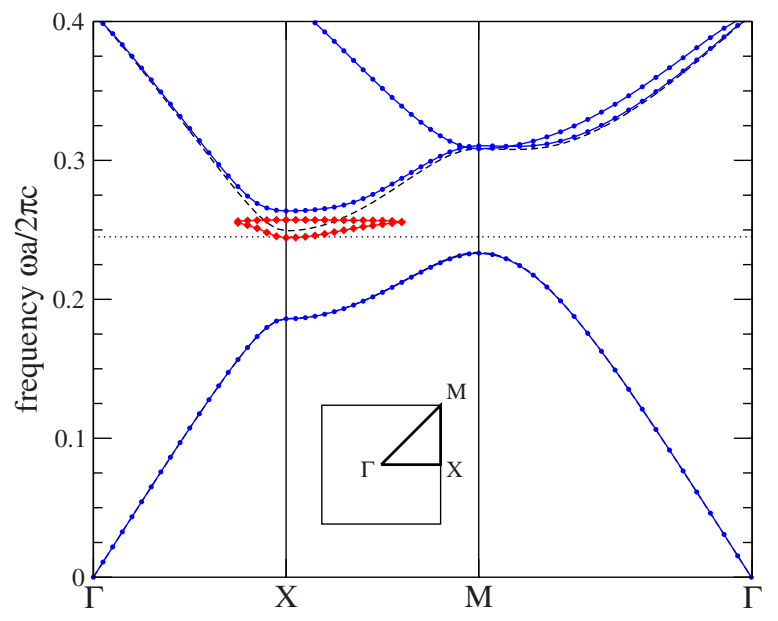

FIG. 9. (Color online) Photonic band structure for the model system with quantum-dot concentration $\eta=0.03$ and when material absorption is ignored (solid lines with symbols). The anomalous dispersion of the pore dielectric constant near the band edge of the second band leads to the formation of propagating modes (lines with diamonds) inside the photonic band gap of the undoped system. For reference, the photonic band structure of the undoped PC is indicated by dashed lines. The horizontal dotted line again depicts the resonance frequency of isolated quantum dots.

similar effect to occur. Furthermore, the geometric interpretation of this effect in terms of a fix-point problem (Sec. III B) suggests that the effect can equally well be realized for TE-polarized radiation in $2 \mathrm{D} \mathrm{PCs}$ as well as in onedimensional and 3D PCs.

While these results of the extended band structure method provide a physical understanding of the interplay of anomalous material dispersion and photonic band structures, they do not contain any information about the effect of the-by the Kramers-Kronig relations unavoidable - material absorption. In particular, it is not clear whether this effect could be observed for realistic experimental parameters.

Therefore, we proceed to a complete analysis of the model system and discuss the frequency-dependent attenuation length. As alluded to in Sec. III, this may be facilitated both through the Wannier function method or the scatteringmatrix approach. In Fig. 10, we display and compare the results of all three methods applied to the model system with quantum-dot concentration $\eta=0.03$ for wave vectors oriented along the crystallographic $\Gamma-X$ direction. In the left panel of Fig. 10, we compare the real part of the wave vector's modulus of the Wannier function approach with the results of the extended band structure technique. Clearly, the extended band structure method cannot obtain a wave-vector solution for frequencies in the stop gap. For the frequencies of the propagating modes, the agreement is very good. Here, we would like to recall that, in contrast to the extended band structure method, the Wannier function approach fully includes material absorption. In the middle panel, we display the imaginary part of the wave vector's complex magnitude as obtained from the Wannier function method. In the undoped reference system, we would expect that this imaginary part is zero for frequencies in the bands, takes on small val- 


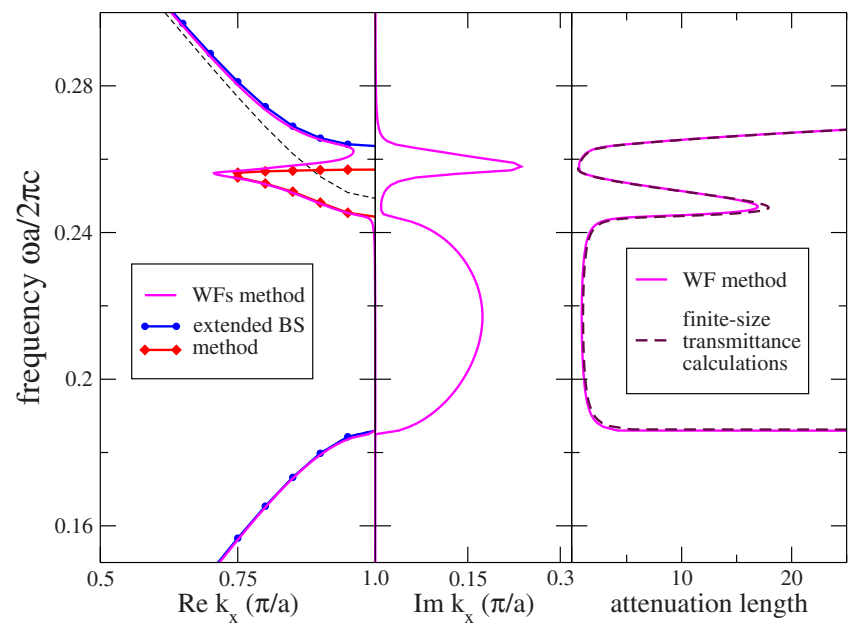

FIG. 10. (Color online) Band structure and attenuation length calculations for the model system with a quantum-dot concentration $\eta=0.03$. In the left panel, Wannier function (WF) calculations are compared to the extended band structure (extended BS) method. The Wannier function calculations yield both real and imaginary parts of the wave vector (middle panel). The latter can be converted into an attenuation length and can be compared with results from transmittance calculations through finite-sized PCs via the scattering-matrix method (right panel). The large values of the attenuation length for frequencies close to the bubble suggest that this effect can be observed experimentally.

ues for frequencies slightly inside the photonic stop band (weak attenuation) and, finally, takes on large values for frequencies near the center of the stop gap (strong attenuation). Material absorption would only modify the actual values but not the qualitative behavior. However, owing to the anomalous material dispersion, we find very low values of this imaginary part for frequencies near the bubble. Finally, in the right panel of Fig. 10, we convert these values into attenuation lengths and compare them with the results of corresponding scattering-matrix computations. Besides a very good agreement between the two methods, we find that the values of the attenuation length for frequencies near the bubble are in excess of ten lattice constants. This suggests that transmittance experiments on 2D macroporous silicon $\mathrm{PCs}^{9}$ should be able to directly observe this (unique) signature of the bubble.

The shape of the bubblelike structure created inside the photonic band gap can be controlled through the parameters entering Eq. (2). For instance, different two-level systems with different resonance frequencies, oscillator strengths, and damping constants could be implanted into appropriate PC backbones. In these cases, considerations similar to our discussion above about the overlap of the targeted mode profile with the dopants' location would have to be carried out.

In the experimental realization via colloidal quantum dots that we have envisaged, the easiest tuning parameter might be the concentration $\eta$ of two-level systems. In Fig. 11, we depict the evolution of the bubblelike structure and the attenuation length of the corresponding modes as the concentration $\eta$ increases. The extension of the bubble both in wave-vector space and in frequency is strongly modified.

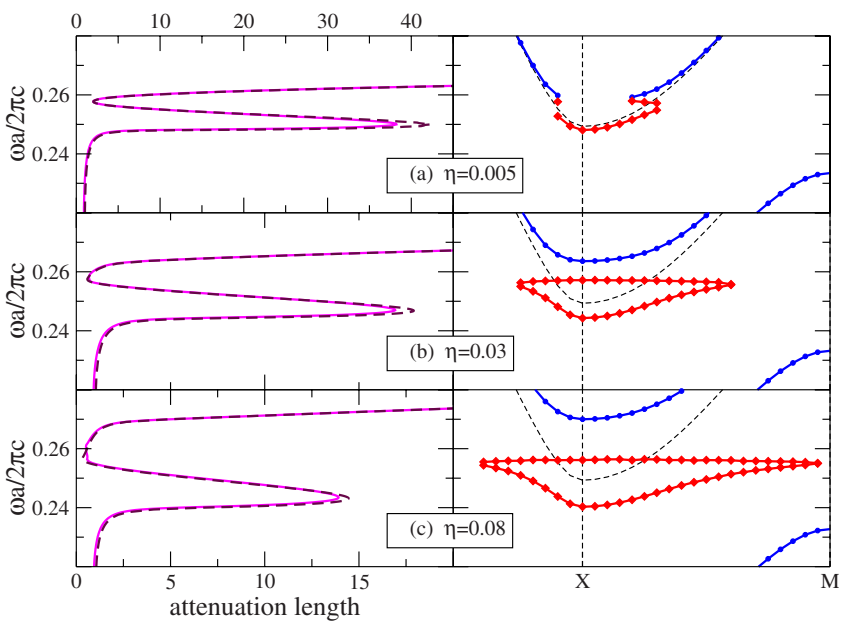

FIG. 11. (Color online) Right panel: evolution of the bubble (lines with diamonds) when the concentration $\eta$ of quantum dots in the model system is increased. The dashed lines depict the band structure of the undoped PC. Left panel: Wannier function (solid lines) and scattering-matrix (dashed lines) calculations of the corresponding attenuation lengths.

Higher concentrations $\eta$ allow a larger wave-vector and frequency range to be covered. Furthermore, these results suggest an alternative interpretation of the bubble formation. Starting from a very low concentration of quantum dots, we observe the onset of a "pinch-off" effect near the band edge. For higher concentration, the bubble is completely separated (completely pinched off) from the original band edge so that, effectively, a stop band is formed. Whether or not this stop band turns into a complete photonic band gap depends on the other regions of wave-vector space, which are less affected by the anomalous dispersion. This stop band is, however, somewhat ill defined. This stems from the fact that the top portion of the bubble, i.e., the flat part of the dispersion relation, corresponds to the unstable branch of the anomalous dispersion and, therefore, exhibits rather large values of the imaginary part of the wave vector. It thus becomes problematic to identify the lower frequency edge of the stop band. In addition, for other materials that exhibit a two-level resonance structure in their dielectric behavior such as systems for which interband transitions are of importance, there does not exist a simple analog to the change of quantum-dot concentration. Therefore, we prefer the first interpretation that anomalous dispersion may lead to additional propagating modes inside the photonic band gap of the undoped system.

At any rate, the resulting tunability of the wave vector and frequency extent of the bubble structure may be beneficial for efficient realizations of superprism effects and other super-refractive phenomena. ${ }^{32}$ Specifically, it might be advantageous to avoid unwanted diffraction effects by limiting the available wave vectors, while simultaneously allowing only narrow frequency bands to couple into the PC. Equally well, one might conceive a modulator via tunable attenuation.

\section{SUMMARY}

We have investigated photonic band structures for PCs whose constituent materials exhibit anomalous dispersion. In 
such systems, anomalous dispersion leads to the occurrence of unconventional propagating modes in the gap. The solution of the corresponding nonlinear eigenvalue problem through an iterative approach allows a simple geometric interpretation of the fact that the limited frequency range over which anomalous dispersion occurs confines these additional modes to a limited wave-vector range in the band diagrams. For the calculation of attenuation lengths associated with these modes, we have developed an efficient on-shell methodology based on photonic Wannier functions. The results of this approach agree very well with those from corresponding transmittance computations and suggest that these modes should be experimentally observable. PCs that exhibit such effects may be realized by infiltrating colloidal quantum dots in a polymer suspension suitably engineered into macroporous silicon PC structures. This technique has been demonstrated. ${ }^{10,11}$ The resulting tunability of PC structures with dispersive components may lead to an enhanced flexibility in tailoring photonic dispersion relations and associated phenomena such as super-refractive effects.

\section{ACKNOWLEDGMENTS}

We acknowledge the support by the DFGForschungszentrum Center for Functional Nanostructures (CFN) of the Deutsche Forschungsgemeinschaft (DFG) at the Universität Karlsruhe within Project A1.1. The work of K.B. and A.G.-M. has been supported by the DFG under Projects Bu 1107/2-2 and Bu 1107/2-3 (Emmy-Noether program).
*Present address: Department of Physics, Iowa State University, Ames, IA 50011, USA.

†resent address: Stepanov Institute of Physics of the National Academy of Sciences of Belarus, 68 Prospekt Nezavisimosti, 220072 Minsk, Belarus.

Present address: Instituto de Microelectrónica de Madrid, Consejo Superior de Investigaciones Científicas, Isaac Newton 8, Tres Cantos, 28760 Madrid, Spain.

${ }^{1}$ E. Yablonovitch, Phys. Rev. Lett. 58, 2059 (1987).

${ }^{2}$ S. John, Phys. Rev. Lett. 58, 2486 (1987).

${ }^{3}$ K. Busch, G. von Freymann, S. Linden, S. F. Mingaleev, L. Tkeshelashvili, and M. Wegener, Phys. Rep. 444, 101 (2007).

${ }^{4}$ P. Halevi and F. Ramos-Mendieta, Phys. Rev. Lett. 85, 1875 (2000).

${ }^{5}$ V. Kuzmiak, A. A. Maradudin, and F. Pincemin, Phys. Rev. B 50, 16835 (1994).

${ }^{6}$ K. Busch and S. John, Phys. Rev. Lett. 83, 967 (1999).

${ }^{7}$ J. B. González-Díaz, A. García-Martín, G. Armelles, D. Navas, M. Vázquez, K. Nielsch, R. B. Wehrspohn, and U. Gösele, Adv. Mater. (Weinheim, Ger.) 19, 2643 (2007).

${ }^{8}$ K. Busch, S. F. Mingaleev, A. García-Martín, M. Schillinger, and D. Hermann, J. Phys.: Condens. Matter 15, R1233 (2003).

${ }^{9}$ A. Birner, R. B. Wehrspohn, U. Gösele, and K. Busch, Adv. Mater. (Weinheim, Ger.) 13, 377 (2001).

${ }^{10}$ M. Steinhart, J. H. Wendorff, A. Greiner, R. B. Wehrspohn, K. Nielsch, J. Schilling, J. Choi, and U. Gösele, Science 296, 1997 (2002).

${ }^{11}$ S. Richter, M. Steinhart, H. Hofmeister, M. Zacharias, U. Gösele, N. Gaponik, A. Eychmüller, A. L. Rogach, J. H. Wendorff, S. L. Schweizer, A. von Rhein, and R. B. Wehrspohn, Appl. Phys. Lett. 87, 142107 (2005).

${ }^{12}$ C. F. Bohren and D. R. Huffman, Absorption and Scattering of Light by Small Particles (Wiley, New York, 1983).

${ }^{13}$ N. W. Ashcroft and N. D. Mermin, Solid State Physics (Saunders,
Philadelphia, 1976).

${ }^{14}$ K. M. Ho, C. T. Chan, and C. M. Soukoulis, Phys. Rev. Lett. 65 , 3152 (1990).

${ }^{15}$ R. D. Meade, A. M. Rappe, K. D. Brommer, J. D. Joannopoulos, and O. L. Alerhand, Phys. Rev. B 48, 8434 (1993).

${ }^{16}$ D. Hermann, M. Frank, K. Busch, and P. Wölfle, Opt. Express 8, 167 (2001).

${ }^{17}$ S. Burger, R. Klose, A. Schädle, F. Schmidt, and L. Zschiedrich, Proc. SPIE 5728, 164 (2005).

${ }^{18}$ J. B. Pendry and A. MacKinnon, Phys. Rev. Lett. 69, 2772 (1992).

${ }^{19}$ N. Stefanou, V. Karathanos, and A. Modinos, J. Phys.: Condens. Matter 4, 7389 (1992).

${ }^{20}$ E. Istrate, A. A. Green, and E. H. Sargent, Phys. Rev. B 71, $195122(2005)$.

${ }^{21}$ J. D. Jackson, Classical Electrodynamics, 3rd ed. (Wiley, New York, 1998).

${ }^{22}$ A. A. Krokhin and P. Halevi, Phys. Rev. B 53, 1205 (1996).

${ }^{23}$ A. Tip, A. Moroz, and J. M. Combes, J. Phys. A 33, 6223 (2000).

${ }^{24}$ K. Busch, A. García-Martín, D. Hermann, L. Tkeshelashvili, M. Frank, and P. Wölfle, Adv. Solid State Phys. 42, 41 (2002).

${ }^{25}$ O. Toader and S. John, Phys. Rev. E 70, 046605 (2004).

${ }^{26}$ D. C. Meisel, M. Diem, M. Deubel, F. Pérez-Willard, S. Linden, D. Gerthsen, K. Busch, and M. Wegener, Adv. Mater. (Weinheim, Ger.) 18, 2964 (2006).

${ }^{27}$ M. G. Moharam, E. B. Grann, D. A. Pommet, and T. K. Gaylord, J. Opt. Soc. Am. A 12, 1068 (1995).

${ }^{28}$ P. Lalanne and G. M. Morris, J. Opt. Soc. Am. A 13, 779 (1996).

${ }^{29}$ G. Granet and B. Guizal, J. Opt. Soc. Am. A 13, 1019 (1996).

${ }^{30}$ D. M. Whittaker and I. S. Culshaw, Phys. Rev. B 60, 2610 (1999).

${ }^{31}$ L. Li, J. Opt. Soc. Am. A 13, 1870 (1996).

${ }^{32}$ H. Kosaka, T. Kawashima, A. Tomita, M. Notomi, T. Tamamura, T. Sato, and S. Kawakami, Phys. Rev. B 58, R10096 (1998). 